Vol. $11(10 / 2009)$

\title{
Ethics of Waste in the Information Society
}

edited by Matthias Feilhauer and Soenke Zehle

\section{Editors of this issue:}

\section{Matthias Feilhauer}

E-Mail: matthias.feilhauer@web.de

Dr. Soenke Zehle

Saarland University, Germany

E-Mail: s.zehle@mx.uni-saarland.de

\section{Editors of IRIE}

\section{Prof. Dr. Rafael Capurro (Editor in Chief),} University of Applied Sciences, Stuttgart, Germany, Wolframstr. 32, D-70191 Stuttgart

E-Mail: rafael@capurro.de

Prof. Dr. Johannes Britz, University of WisconsinMilwaukee, USA University of Pretoria, South Africa E-Mail: britz@uwm.edu

Prof. Dr. Thomas Hausmanninger, University of Augsburg, Germany, Universitätsstr. 10 , D-86135 Augsburg

E-Mail: thomas.hausmanninger@kthf.uniaugsburg.de
Prof. Dr. Makoto Nakada, University of Tsukuba, Japan, Tennodai, Tsukuba, 305-8577 Ibaraki E-Mail: nakadamakoto@msd.biglobe.ne.jp

Dr. Felix Weil, QUIBIQ, Stuttgart, Germany, Heßbrühlstr. 11, D-70565 Stuttgart

E-Mail: felix.weil@quibiq.de

\section{Editorial Office}

\section{Marcus Apel}

Rotebühlstr. 145, D-70197 Stuttgart

E-Mail: MarcusApel@gmx.info 